\title{
Understanding the Impact of Interference on Collaborative Relays
}

\author{
Yan Zhu, Student Member, IEEE and Haitao Zheng, Member, IEEE
}

\begin{abstract}
Collaborative relays achieve the benefits of spatial diversity without requiring physical antenna arrays at the end devices. While many studies have demonstrated its effectiveness in an isolated single source-destination system, applying cooperative relays to a large-scale wireless network remains challenging. We show that a large wireless system with cooperative relays can be penalized by the elevated level of interference it produces. By examining the inter-dependency between interference management and cooperative relay strategies, the penalty is modeled by an increase in spectrum resource usage and translate it into a penalty on single-link throughput. This throughput penalty serves as a reference for designing collaborative relays in isolated scenarios. To mitigate the impact of interference, we present two channel allocation mechanisms for collaborative relay systems with different fairness and utilization tradeoffs. Simulation results confirm our analytical findings and demonstrate the effectiveness of our proposed channel allocation mechanisms.
\end{abstract}

\section{Index Terms}

Wireless network, collaborative relay, interference management, resource allocation.

\section{INTRODUCTION}

Wireless networks suffer from frequent and unpredictable packet losses because of channel fading. Many wireless systems use multiple-input multiple-output (MIMO) antenna arrays which

Manuscript received April 12, 2007; revised November 15, 2007; accepted December 3, 2007.

Yan Zhu is with Dept. of Electrical Engineering and Computer Science, Northwestern University, Evanston, IL 60208 USA. Email: yan-zhu@ northwestern.edu.

Haitao Zheng is with Dept. of Computer Science, University of California, Santa Barbara, CA 93106 USA. Email: htzheng@cs.ucsb.edu. 
exploit spatial diversity to mitigate channel fading and extend transmission range. Most portable wireless devices, however, can carry 1 or 2 antennas only, which in turn, significantly limits the effectiveness of MIMO mechanism.

Recent proposals [1]-[3] address this limitation through collaborative relays. Devices equipped with omnidirectional antennas can intersect a neighboring transmitter's signal and relay it to the designated receiver. The receiver combines multiple signal streams from both source and relay nodes to recover the transmitted signal with higher reliability. By collaboratively relaying the signal, nodes form a virtual antenna array, achieving the same benefits as a MIMO system with only one antenna per device.

There have been significant efforts towards understanding and improving the benefits of collaborative relays in small (isolated) wireless networks. In a simple single source-destinationrelay scenario, existing contributions span from analysis of channel capacity [4], [5] and diversitymultiplexing tradeoff [2], [6], to algorithm design for power allocation [7], [8], relay selection [9] and link retransmission [10]. Others extend the results to a more complex scenario with multiple source-destination-relay nodes, including capacity analysis [11], resource allocation [12], power allocation [8] and the use of group-nulling to increase array gain [13]. Overall, these studies have demonstrated the effectiveness of collaborative relays in a small-scale isolated wireless network.

Deploying collaborative relays in large-scale wireless networks, however, faces many challenges [9], [14], [15]. In addition to requiring efficient protocols for node collaboration, an critical challenge is to address the impact of an elevated interference produced by signal relaying. When interference is a dominant limiting factor, increase in interference level translates into degraded throughput and higher packet losses.

In this paper, we show both analytically and experimentally that the use of collaborative relays in large wireless networks is penalized by the elevated level of interference. We characterize the penalty in terms of increase in the amount of channel resource consumption, and translate it to a throughput penalty in an isolated source-destination-relay scenario. This throughput penalty provides a reference for designing collaborative relay strategies. In particular, we show that a large-scale wireless network can benefit from the use of collaborative relays only if the gain of relaying in isolated scenarios is higher than a critical ratio $T>1$ rather than $T=1$.

In addition to identifying and characterizing the interference penalty, we also propose channel allocation mechanisms to mitigate the impact of interference. We propose to assign orthogonal 
channels to conflicting transmission links. By assigning different priorities to direct and relay links, we design two approaches that trade off fairness against system throughput. We perform extensive simulations to verify our analytical findings and evaluate the performance of proposed channel allocation mechanisms.

The rest of the paper is organized as follows. We start from a brief overview of collaborative relays in Section II, and model the network-wide interference in Section III. In Section IV, we characterize the impact of interference using a conflict-graph based resource allocation problem, and design different channel allocation strategies. In Section V we examine the asymptotic interference condition and analytically derive the penalty of increased interference. In Section VI we perform simulations to evaluate the proposed channel allocation algorithms and validate the theoretical findings. Finally, we discuss design implications and future directions in Section VII and conclude in Section VIII.

\section{BACKGROUND ON COLLABORATIVE RELAY}

Collaborative relays form a virtual multiple antenna system by nodes relaying signals for one another. Existing studies have proposed several relay strategies with different performance and complexity tradeoffs. In this section, we briefly describe a representative set of relay strategies. We focus on the amount of channel resource each node consumes.

\section{A. Collaborative Relay Strategies}

Figure 1 shows a basic collaborative relay system. Using omnidirectional antennas, a transmitter $S$ transmits a signal to its designated receiver $D$. A collaborative neighbor $R$ intersects the signal and forwards it to $D$. $D$ combines two signal streams, $S \Rightarrow D$ and $R \Rightarrow D$ into a single stream which has higher resistance to channel fading and noise, hence, higher probability of being successful decoded. In general, there are two types of collaborative relays [2]:

Amplify and forward (AF). $R$ receives signals from $S$, amplifies and forwards them to $D$ without demodulation or decoding. As a result, it also amplifies the noise associated with link $S \Rightarrow R$. When the time gap between direct and relay paths is small, $D$ can use a RAKE receiver to combine both signal streams [2], [16]. The system behaves like a single link with one transmit antenna and two receive antennas. We refer to this strategy as AF-RAKE. Note that AF-RAKE requires a full-duplex radio at $R$ that can transmit and receive from the same channel 


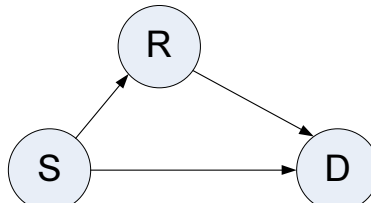

Fig. 1. A simple example of cooperative relays where node $S$ communicates with node $D$ with the help of a collaborative relay $R$. Node $D$ collects signals from both $S$ and $D$, and reproduces the signal at a higher success probability.

TABLE I

SUMMARY OF RELAY STRATEGIES

\begin{tabular}{|c|c|c|c|c|c|}
\hline & No. of channels & No. of time slots & Decode at relay & Duplexity & Throughput \\
\hline AF-RAKE & 1 & 1 & No & Full & $W \cdot C_{A F-R A K E}$ \\
\hline AF-TDD & 1 & 2 & No & Half & $\frac{1}{2} W \cdot C_{A F-R A K E}$ \\
\hline AF-FDD & 2 & 1 & No & $(2 \times)$ Half & $W \cdot C_{A F-R A K E}$ \\
\hline DF & 1 & 2 & Yes & Half & $W \cdot C_{D F}[2]$ \\
\hline
\end{tabular}

concurrently. A low-cost alternative is AF-FDD where $R$ receives from $S$ on one channel and forwards the signal on a different channel, while $D$ receives from both channels and combines the signals. This requires $R$ and $D$ to use two half-duplex radios and two channels. When only a single half-duplex radio is available at each node, the system can multiplex $S \Rightarrow D$ and $R \Rightarrow D$ into two time slots, referred to as AF-TDD.

Decode and forward (DF). In this approach, $R$ decodes the signal from $S$, re-encodes it with a sophisticated coding technique [2], [5] and forwards the re-generated signal to $D$. Since $R$ has to decode and re-encode the signal, $S \Rightarrow D$ and $R \Rightarrow D$ are carried out in two time slots. Note that this operation only requires a half-duplex radio at $R$ and $D$ and one channel.

Table I summarizes each strategy and its resource usage. Note that DF has the same resource consumption as that of AF-TDD. In this paper, we focus on $A F$ strategies to examine the system performance of collaborative relay, rather than proposing any new coding and transmission technologies. However, our work can be easily extended to include other relay strategies.

\section{B. Capacity Abstractions}

Next, we utilize the information theoretical capacity to characterize per-link transmission efficiency of collaborative relays. The capacity of a transmission represents the maximum amount of 
information per second per Hertz that can be sent successfully. It provides a good approximation of link efficiency in real wireless systems without delving into complex coding, detecting and decoding procedures. In this paper, we use ergodic and outage capacities to characterize a wireless fading channel, use throughput which is the product of the channel capacities and spectrum bandwidth to characterize the transmission quality on the channel. Next, we describe these two capacities in details.

Ergodic capacity represents the expectation of the instantaneous channel capacity, averaged across channel fading. It captures the average packet throughput for delay non-sensitive applications. We can approach the ergodic capacity by adapting transmission settings (e.g. power, modulation, coding rate) to channel variations.

Outage capacity measures the maximum data rate supported with a probability of $1-\zeta$ assuming a fixed transmission rate, where $\zeta$ is the outage probability or the maximum tolerable packet loss rate. An outage occurs if the transmission rate is higher than the instant channel capacity, (e.g., no packet can be sent successfully). Different from the ergodic capacity, the outage capacity captures the maximum supported data rate for delay sensitive applications such as VoIP and video streaming.

In general, there are no close-form expressions of ergodic and outage capacities. The work in [2] provides simplified approximations of both capacities assuming each node transmits at a constant power and experiences independent frequency flat fading. Let $\gamma$ represent the transmit signal-to-noise ratio and $W$ represent the spectrum bandwidth of each channel, we have the capacities of direct transmission and AF-RAKE as follows:

Ergodic Capacity:

$$
\begin{aligned}
C_{E, D T}= & E_{h_{S D}(t)} \log \left(1+\left|h_{S D}(t)\right|^{2} \gamma\right) \\
C_{E, A F-R A K E}= & E_{h} \log \left(1+\left|h_{S D}(t)\right|^{2} \gamma+\right. \\
& \left.f\left(\left|h_{S R}(t)\right|^{2} \gamma,\left|h_{R D}(t)\right|^{2} \gamma\right)\right)
\end{aligned}
$$

where

$$
f(x, y):=\frac{x y}{x+y+1}
$$




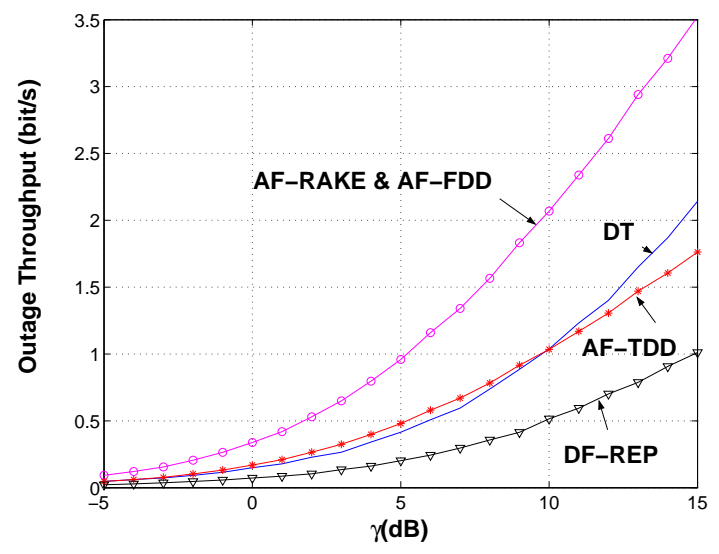

(a) Outage $(\zeta=10 \%)$ Throughput

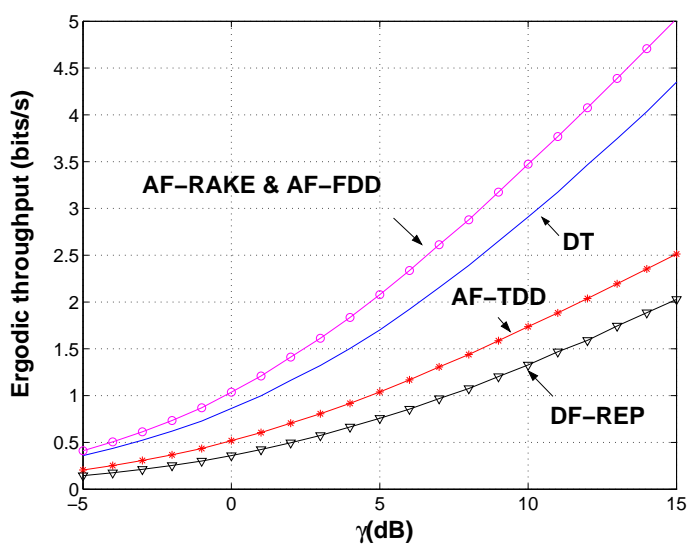

(b) Ergodic Throughput

Fig. 2. Throughput of different cooperative strategies for $W=1$.

\section{Outage Capacity at an outage probability of $\zeta$ :}

$$
\begin{aligned}
C_{O, D T} & =\log \left(\gamma \zeta \sigma_{s, d}^{2}+1\right) \\
C_{O, A F-R A K E} & =\log (\gamma \sqrt{\zeta \phi}+1)
\end{aligned}
$$

where $\frac{1}{\phi}:=\frac{1}{2 \sigma_{s, d}^{2}} \frac{\sigma_{s, r}^{2}+\sigma_{r, d}^{2}}{\sigma_{s, r}^{2} \sigma_{r, d}^{2}}$ and $\sigma_{i, j}^{2}:=E\left(\left|h_{i j}(t)\right|^{2}\right) . h_{i j}(t)$ represents the channel response from node $i$ to $j$ at time $t$ that captures the effects of path-loss, shadowing and fading.

Table I provides a summary of various relay strategies. Note that the capacities of AF-TDD are half of those of AF-RAKE since AF-TDD splits the transmissions into two time slots. The throughput of AF-FDD are the same as those of AF-RAKE assuming that in AF-RAKE, nodes 
$S, R$ and $D$ all operate on a single channel of bandwidth $W$, while in AF-FDD, they operate on two channels, each of bandwidth $W$. Therefore, per-band throughput of AF-FDD is half of that of AF-RAKE. Figure 2 plots the throughput of different strategies as a function of $\gamma$, assuming $W=1$ and all channels follow Rayleigh fading with $\sigma_{i, j}^{2}=1$. We see that AF-RAKE and AF-FDD significantly outperform direct transmission; and AF-TDD is slightly beneficial in terms of outage throughput for small signal-to-noise ratio. Overall, AF schemes achieve higher performance improvement in terms of outage capacity. This is because transmitting via two independent paths can significantly improve packet delivery rate. For more details about ergodic and outage capacities, please see [17, Ch. 5] and [2].

\section{Modeling Collaborative Relay in Ad hoc Networks}

While results in Figure 2 demonstrate the effectiveness of collaborative relays in a network of three nodes, deploying them in a large wireless network must consider the impact of elevated interference from relaying. In this section, we present an analytical model on the network-wide interference. We start from describing two general interference models, and extend them to collaborative relays based networks.

\section{A. General Interference Model}

We consider a large-scale network with static nodes and single-hop transmissions. We use two receiver-driven interference models, the Physical Model and the Protocol Model, to characterize radio transmissions in the presence of interference. The physical model was introduced in [18] to integrate the impact of channel fading while the protocol model is a simplified alternative [19], [20].

Physical Model. Let $\mathbb{S}$ denote the set of wireless nodes that communicate on the same channel ${ }^{1}$. When node $i^{\prime}$ communicates to node $i$, the received signal-to-interference-noise ratio at node $i$ is defined as

$$
\begin{aligned}
& S I N R_{i}(t)=\frac{P_{i^{\prime}}\left|h_{i i^{\prime}}(t)\right|^{2} d_{i i^{\prime}}^{-\alpha}}{\sigma^{2}+I_{i}(t)}, \\
& I_{i}(t)=\sum_{j \in \mathbb{S}, j \neq i^{\prime}} P_{j}\left|h_{i j}(t)\right|^{2} d_{i j}^{-\alpha} .
\end{aligned}
$$

\footnotetext{
${ }^{1}$ We assume each source only communicates with one destination node and vice versa.
} 
where $P_{j}$ is the transmit power at neighboring node $j, \sigma^{2}$ represents the thermal noise variance, $h_{i j}(t)$ represents the small-scale channel fading from transmitter $j$ to receiver $i$ at time $t$, and $d_{i j}$ is the distance between $i$ and $j$. The large-scale fading (path loss) is approximated by $d_{i j}^{-\alpha}$ where $\alpha=2$ for free space propagation and $\alpha>2$ for general environments [18], [21]. Each link's transmission can be successfully decoded only if its received $S I N R$ is above a given threshold $S I N R_{0}$ for a given transmission rate. Therefore, at each time instance, the total level of tolerable interference at node $i$, defined by $I_{0, i}$ is

$$
I_{0, i}(t)=\frac{P_{i^{\prime}}\left|h_{i i^{\prime}}(t)\right|^{2} d_{i i^{\prime}}^{-\alpha}}{S I N R_{0}}-\sigma^{2}
$$

Given a set of transmissions in $i$ 's neighborhood, only if $I_{i}(t) \leq I_{0, i}(t)$, $i^{\prime}$ can successfully communicate to $i$ at a particular rate determined by $S I N R_{0}$.

Protocol Model. While providing a sophisticated model of interference, the physical model also requires extensive knowledge of channel instance $h_{i j}$, which is computational-expensive to obtain. A simplified version is to use a no-talk distance $r_{I}$. Assuming each user transmits at a constant power $P$, for each receiver $i$, if all the transmitters except the node $i^{\prime}$ are more than $r_{I}(i)$ distance away, then with very high probability that the overall accumulated interference is less than the tolerable threshold, i.e.

$$
\operatorname{Prob}\left\{I_{i}(t) \leq I_{0, i}(t)\right\}>1-\epsilon
$$

where $\epsilon \ll 1$ represents tolerable packet loss rate. In another word, $r_{I}(i)$ is a location-dependent distance-driven interference-sensitive range where if any transmitter is within $r_{I}(i)$ distance from $i$, it could potentially interfere with $i$ 's reception. Note that the value of $r_{I}(i)$ depends on the level of transmit power $P$, transmit power density, radio propagation condition, transmission frequency, $S I N R_{0}$ and $\epsilon$. Assuming negligible background noise, $r_{I}(i)$ has a simple lower bound [20]:

$$
r_{I}(i) \geq S I N R_{0}^{\frac{1}{\alpha}} d_{i i^{\prime}}
$$

In order to applying the general interference models to large-scale wireless networks, we make the following assumptions:

(1) Each link's interference sensitive region is the combination of two end nodes. Because transmissions between any two nodes require handshaking, e.g. RTS-CTS-DATA-ACK in IEEE 802.11, each node of a link pair becomes both a transmitter and receiver, i.e. a transceiver. Any 
transmitter within their interference-sensitive ranges could degrade the reception. Therefore, interference-sensitive region of a link is the combination of those of both individual nodes, as illustrated by Figure 3(a). We note that any node inside the interference-sensitive region of a link will potentially interfere with the transmission.

(2) We only focus on the protocol model because of its simplicity. While the physical model provides accurate modeling of interference, applying it to a large scale system is computationalexpensive. Even as a pessimistic binary simplification of the physical model, the protocol model captures the essence of accumulated interference from neighboring transmitters as well as the impact of radio propagation model, without delving into the details of demodulation, decoding and signal processing.

(3) We assume nodes transmit at a fixed power. Instead of adjusting transmit power level to reduce interference, we focus on using spectrum redundancy to assign transmissions that could possibly interfere one another with orthogonal non-interfering channels or time slots, while allowing spectrum reuse between links that are well-separated.

With these assumptions in mind, we now describe the interference model for cooperative relays.

\section{B. Collaborative Relay Interference Model}

Physical Model. Let $\mathbb{S}$ and $\mathbb{R}$ denote the sets of source nodes and the relay nodes that communicate on the same channel, respectively. Assume no nodes can play two roles, e.g. $\mathbb{S}$ and $\mathbb{R}$ are disjoint. A transmission from $i^{\prime}$ to $i$ which is assisted by an amplify-and-forward collaborative relay, $l$, has a received $S I N R$ of

$$
\begin{aligned}
& \operatorname{SINR}_{i}(t) \\
= & \frac{P_{i^{\prime}}\left|h_{i i^{\prime}}(t)\right|^{2} d_{i i^{\prime}}^{-\alpha}+P_{l} \frac{\operatorname{SINR}_{l}(t)}{\operatorname{SINR}_{l}(t)+1}}{\sigma^{2}+\sum_{j \in \mathbb{S} \cup \mathbb{R}, j \neq i^{\prime}, j \neq l} P_{j}\left|h_{i j}(t)\right|^{2} d_{i j}^{-\alpha}+P_{l} \frac{1}{\left(\operatorname{SIN} R_{l}(t)+1\right)}}
\end{aligned}
$$

where

$$
S I N R_{l}(t)=\frac{P_{i^{\prime}}\left|h_{l i^{\prime}}(t)\right|^{2} d_{l i^{\prime}}^{-\alpha}}{\sigma^{2}+\sum_{j \in \mathbb{S} \cup \mathbb{R}, j \neq i^{\prime}} P_{j}\left|h_{l j}(t)\right|^{2} d_{l j}^{-\alpha}}
$$

Note that for AF, relay node $l$ amplifies its perceived interference to node $i$. Therefore, it is equally important to prevent interference at relay and destination nodes. At the same time, relay 
node $l$ extends the interference range of the direct transmission. These observations lead us to re-define the interference-sensitive regions of collaborative relays in the protocol model.

Protocol Model. Following the general protocol model, we approximate the interferencesensitive region of a collaborative relay as the combination of interference regions of source, relay and destination nodes, as shown in Figure 3(b). Overall, each transmission (direct or relay) has an interference-sensitive region which is the combination of the regions associated with the nodes involved. Any transmitter in the sensitive region will produce non-negligible interference to the receiver and degrade its performance. From this model, we see that a collaborative relay has much larger interference-sensitive region, and also produces additional interference to peers. Such increased interference sensitivity will lead to a penalty in system performance, which we will examine in the following section.

\section{Resource Allocation for Collaborative Relays}

In this section, we study the penalty of elevated interference by examining the amount of spectrum resource required to mitigate interference in collaborative relay networks. While prior work focuses on applying power control to reduce interference, we take a different approach to utilize spectrum redundancy. Assuming a multi-channel system, we focus on channel allocations that assigns orthogonal channels to conflicting links while allowing well-separated links to reuse channels ${ }^{2}$.

To characterize the penalty, we compare the amount of spectrum resource each link can get with and without using relays. We start from modeling the corresponding resource allocation problem into a conflict-graph based graph-coloring problem, and then propose two allocation mechanisms.

\section{A. Reduction to Conflict Graph and Graph Coloring}

We show that the network-wide interference can be modeled by a conflict graph. We introduce a graph $G(V, E)$ with vertices $V$ and undirected edges $E$ [22]. Each vertex $u \in V$ represents a link rather than a single communication node. An edge exists between two link-vertices $u$ and $v$

\footnotetext{
${ }^{2}$ Note that the concept of channels is logical as a channel can be a physical frequency band, a time slot, or a channelization code.
} 


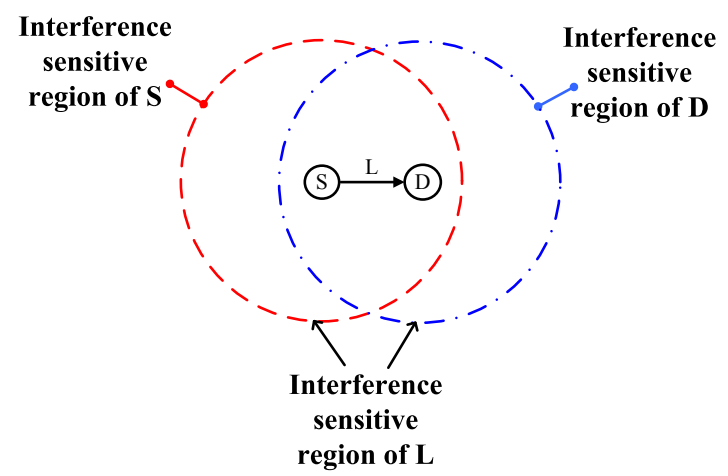

(a)

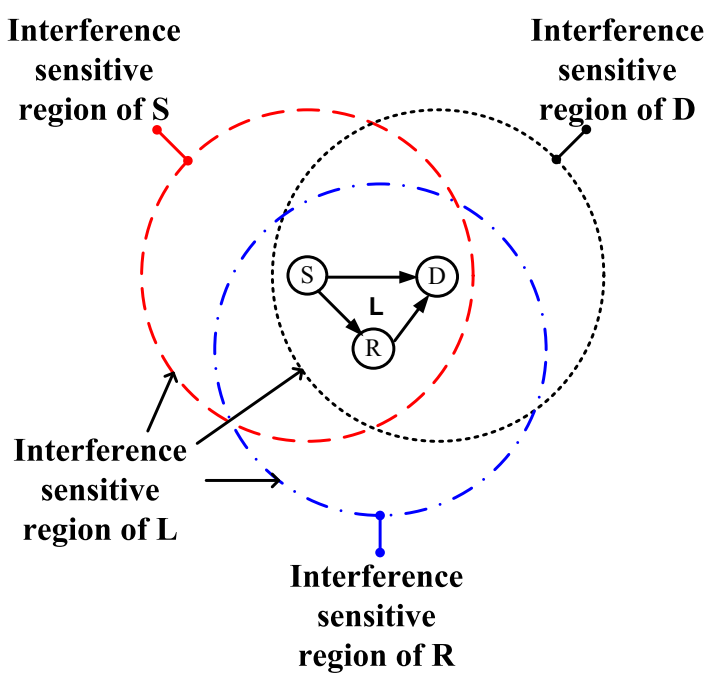

(b)

Fig. 3. Interference-sensitive regions of (a) a direct link (b) a collaborative relay

if they conflict and must use different channels. Assuming symmetric interference patterns, the edges are undirected.

In Figure 4, we illustrate the procedure to build a conflict graph from a network topology.

1) Direct Transmissions: Each link maps to a vertex in the conflict graph. Any two vertices connect if one falls into the other's interference-sensitive region.

2) AF-RAKE: Combine both the direct and relay links into a single vertex because they operate on the same channel. For example, in Figure 4, we combine the node $S 1, R 1, D 1$ and their associated links into a single vertex $(L 1)$.

3) AF-FDD: The direct and relay links use different channels and hence map to two vertices. The edges should be constructed similarly as previous two cases. 


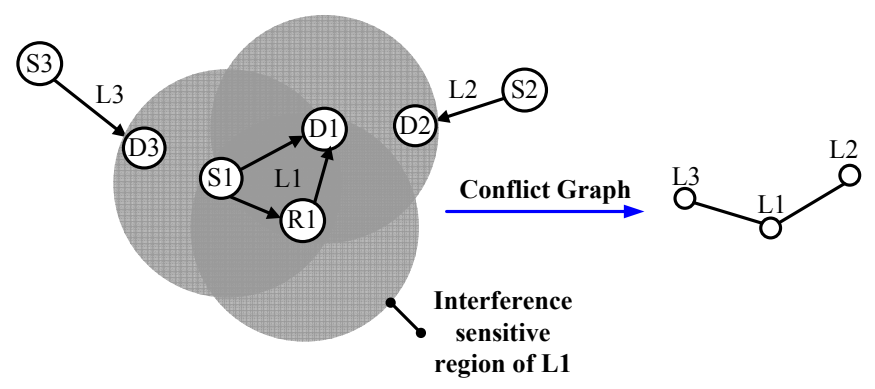

Fig. 4. An Example of Conflict Graph

For simplicity, we assume a network with $M$ channels of equal bandwidth, slow-varying network topology and traffic volume. The resource allocation problem is how to assign channels to links so that they do not conflict. Because of the interference constraints, this problem is known to be NP-complete [23].

\section{B. Allocating Channels among Direct Transmissions}

Existing study converts channel allocation problems into graph coloring problems by mapping each channel to a color [24], [25]. The coloring algorithm is constrained by that if an edge exists between any two vertices, they can not simultaneously use one color. While the general coloring problem is known to be NP-complete [23], existing work shows that heuristic based approaches produce good approximations to the optimal solution. When the number of channels $M$ is adjustable, the algorithms in [24] can be applied to derive the minimum number of channels required for each link to obtain a channel, i.e. to maximize per-channel bandwidth $1 / M$. When the number of channels is fixed, the algorithms in [25] can be applied to maximize the number of links being assigned with a channel. Distributed algorithms such as local bargaining and rule guided self-adjustment [26], [27] provide good approximations to the centralized graph coloring approaches.

The performance of channel allocation depends on the maximum vertex degree of the conflict graph. The degree of a vertex is the number of vertices which is connected to, i.e. the number of conflicting neighbors. The number of channels required to color a conflict graph is upper bounded by the maximum vertex degree +1 [22]. Compared to direct transmissions, collaborative relays result in conflict graphs with higher vertex degree and hence require more channels to achieve conflict-free transmissions. In other words, when the number of channels is small, some links 
might be disabled due to lack of channels.

\section{Allocating Channels among Collaborative Relays}

We extend the algorithms in [25] to distribute channels among transmission links. Using AFRAKE, we assign the same channel to both the direct link $S \Rightarrow D$ and the relay link $R \Rightarrow D$. Using AF-FDD, we assign two different channels to these links. However, when the number of channels is small and hence some links are blocked, we need to choose the links intelligently to maximize system utility.

Next, we present two different allocation strategies that assign different priorities to direct and relay links.

Collaborative Allocation (CA). This allocation scheme prioritizes direct links over relay links by performing allocation sequentially starting from direct links. The system first allows direct links to negotiate for channel usage via schemes in [25], [26]. Only direct links with channel allocated can set up their relay links. Using AF-RAKE, a relay is set up only if the channel assigned to the direct link is available at the relay link, i.e. it does not conflict with neighboring links with channel assigned. Using AF-FDD, a relay is set up only if a channel different from that of the direct link is available. Overall, using such sequential assignment, CA scheme is fairness-driven, i.e. it attempts to minimize the impact of relay links on direct links. Although minimizing the probability of blocking direct transmissions, this strategy may not be the optimal strategy for maximizing system throughput.

Non-collaborative Allocation (NCA). This scheme performs channel allocation at the transmission set level. A collaborative relay set consists of both direct and relay links. For AF-RAKE, each set is assigned with one channel. If there is no channel available, both links are disabled. For AF-FDD, each set is assigned with two channels. If there is no channel available, both links are disabled. If there is only one channel available, only the direct link will be set up. This scheme attempts to maximize system throughput at the cost of blocking some direct links.

In addition to using conflict-free channel allocation, we can also implement CA and NCA strategies using channel contention protocols. In multi-channel MAC protocols, links contend for channels. CA can assign higher priority in terms of shorter contention window, while NCA requires both direct and relay links contend as a set. 


\section{ASymptotic Analysis of Collaborative Relays}

In this section, we analyze the effectiveness of collaborative relays in large-scale wireless networks. We explore the asymptotic trend assuming a large-scale random wireless network where each node communicates with one of its farthest neighbors at the edge of communication range. We reduce the network into a unit disk graph, and model the penalty of expanded interference by an increase in channel resource usage, and translate it into a penalty of single-link capacity. This penalty can be used to design cooperative relay strategy that takes into account the impact of interference.

\section{A. Unit Disk Graph Model}

We start from a brief discussion on the unit disk graph model used for our analysis. Assuming a network of $n$ randomly deployed nodes, we can build a unit disk graph as follows: each node maps to a vertex, two vertices connect if and only if they are within a distance of $d$. An alternative way to build this graph is to associate each node with a disk which is centered on the node and with radius $d / 2$. Two vertices connect if and only if the two corresponding disks overlap. A simple geometric argument can justify the equivalency between these two constructions. Additional information on unit disk graph can be found in [28]. Next, we show that one can approximate the interference condition of a collaborative relay network by a unit disk graph.

\section{B. A Simplified Model of Large-scale Random Networks}

In Section IV, we show that each collaborative relay or direct transmission has an interference sensitive region. However, the size of the disk depends on the distance between source, relay and destination nodes. To make the analysis tractable, we consider the worst case scenario: each transmission pair is of the maximum distance apart. This produces the largest interference sensitive region. Again, we assume that each node has the same interference-sensitive range $r_{I}$.

Under these assumptions, we show that one can model the direct transmission and the collaborative relay transmission using a unit disk graph. To do so, we first choose the center of the link as the disk center. We approximate the interference sensitive region with a large disk of radius $X$, and the final unit disk has a radius of $X / 2$, which follows from the second construction of unit 
disk graph described in Section V-A. Next we illustrate the process for both direct transmission and collaborative relay transmission.

In a direct transmission, source node $S$ and destination node $D$ are of distance $r_{C}$ apart, where $r_{C}$ is the communication range. For any two links, if their median are $r_{I}+r_{c}$ apart, then they will not conflict. Therefore, we can approximate the interference-sensitive region of link $S-D$ by an open disk with radius $d_{d}$.

$$
d_{d}\left(r_{C}\right)=r_{I}+r_{C}=(\lambda+1) r_{C}
$$

where $\lambda=r_{I} / r_{C}$, hereby referred to as the interference sensitive ratio. Thus, the disk associated with $S$ - $D$ link has radius of $d_{d} / 2$.

In the corresponding conflict graph, each link $(S, D)$ is represented by a disk of radius $d_{d} / 2$ centered at the median location between $S$ and $D . d_{d}$ represents the maximum separation between the centers of two disks where each transmission won't be disrupted by any transmitter of the other disk. Any two links could conflict if their corresponding disks overlap, i.e. the centers of both disks are no more than $d_{d}$ apart.

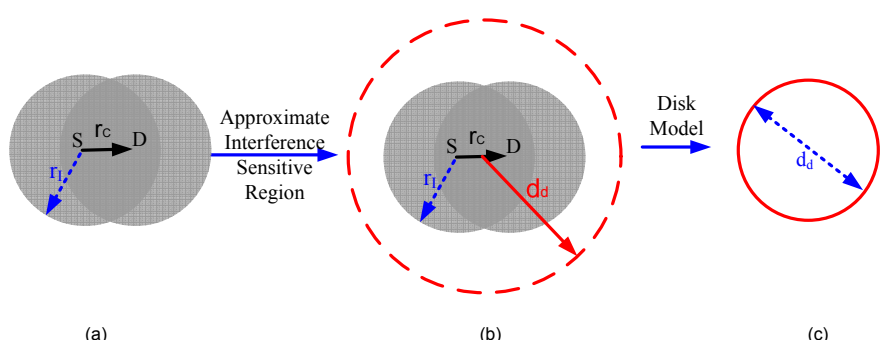

Fig. 5. Disk Model of Direct Transmission. (a) Interference-sensitive region of direct transmission. (b) Approximation of the region by a large disk. (c) Disk associated with a direct transmission in disk graph model.

Similarly, when $S$ and $D$ are assisted by a relay node $R$ where $d(S, R)=r_{1}, d(R, D)=r_{2}$, 
we can approximate the interference-sensitive region as a disk of radius ${ }^{3}$

$$
\begin{aligned}
& d_{\text {coop }}\left(r_{C}, r_{1}, r_{2}\right) \\
= & \begin{cases}r_{I}+r_{C} & : r_{1}^{2}+r_{2}^{2} \leq r_{C}^{2} \\
r_{I}+\frac{1}{2} r_{C}\left(\sqrt{1+\frac{\left(r_{1}^{2}+r_{2}^{2}-r_{C}^{2}\right)^{2}}{4 r_{1}^{2} r_{C}^{2}-\left(r_{C}^{2}+r_{1}^{2}-r_{2}^{2}\right)^{2}}}+1\right) & : \text { Otherwise }\end{cases}
\end{aligned}
$$

Apparently, the interference-sensitive region depends on choice of $r_{1}$ and $r_{2}$. We focus on the worst-case interference scenario where the relay node is of distance $\rho r_{C}$ from both the source and destination, where $\rho$ is referred to as relay distance factor. ${ }^{4}$ Let $r_{1}=r_{2}=\rho r_{C}$ and we can approximate the interference-sensitive region of the relay link with an open disk of radius $d_{\text {coop }}$,

$$
d_{\text {coop }}= \begin{cases}(\lambda+1) r_{C} & : \quad 1 / 2<\rho<\sqrt{2} / 2 \\ \left(\lambda+\frac{\rho^{2}}{2 \sqrt{\rho^{2}-1 / 4}}+\frac{1}{2}\right) r_{C}: & \sqrt{2} / 2 \leq \rho \leq \lambda\end{cases}
$$

As a result, the disk associated with the cooperative relay link is centered at the median of $S, D$, and $R$ and with radius $d_{\text {coop }} / 2$.

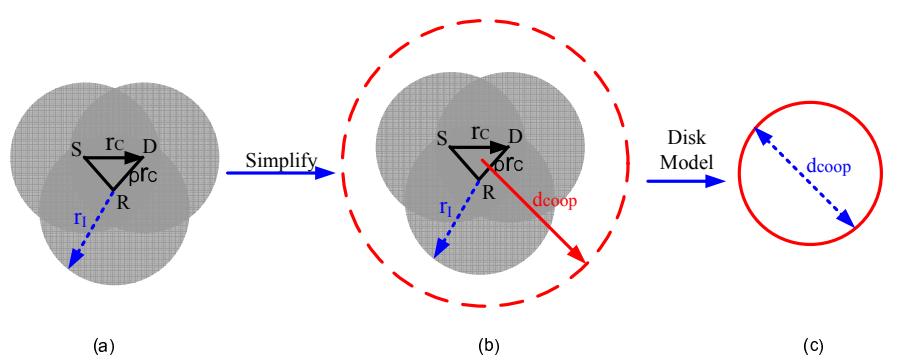

Fig. 6. Disk Model of Collaborative Relay. (a) Interference-sensitive region of a cooperative relay. (b) Approximation of the region by a large disk. (c) Disk associated with a cooperative relay in disk graph model.

Using the above disk model, we can characterize a large random network using a random disk graph. Each vertex represents a randomly deployed link (direct or collaborative relay), located at the median. Each vertex is associated with a disk of radius $d_{d} / 2$ or $d_{\text {coop }} / 2$ where if two disks overlap, the corresponding vertices conflict and an edge exists between the two. When a

\footnotetext{
${ }^{3}$ We note that in practice, relaying leads to an increase in the transmit power density which directly affects $r_{I}$. For simplicity, we assume that $r_{I}$ remains the same and focus on the expansion of the overall interference sensitivity region.

${ }^{4}$ We assume relay nodes must be fairly close to its source and destination nodes, $\rho \leq \lambda$. On the other hand, $\rho$ must be large enough to allow a sufficient probability of finding a relay.
} 
network contains only direct links or only collaborative relay links, the corresponding random disk graph becomes a random unit disk graph.

\section{Asymptotic Resource Usage of a Random Network}

Using existing results of random unit disk graph [29], we can derive the asymptotic number of channels required to achieve conflict-free transmission.

Theorem 1 (C. McDiarmid [29]): Suppose $n$ disks with diameter $d(n)$ are uniformly distributed in a unit area. Let $d=d(n)$ satisfy $d \rightarrow 0$ and $d^{2} n / \ln (n) \rightarrow \infty$ as $n \rightarrow \infty$. Let $\chi(n)$ represent the minimum number of channels required to assign each disk with a channel without any conflict. Let

$$
k(n)=\frac{\pi}{4} d^{2} n
$$

Then as $n \rightarrow \infty, \chi(n) / k(n) \rightarrow 2 \sqrt{3} / \pi$ almost surely. ${ }^{5}$

We define $\chi_{D}\left(n_{L}\right)$ as the minimum number of channels required when there are $n_{L}$ direct transmission links only, and $\chi_{C}\left(n_{L}\right)$ as the minimum number of channels for $n_{L}$ collaborative relay links only. Because $\chi(n)$ is a random variable, so are $\chi_{D}\left(n_{L}\right)$ and $\chi_{C}\left(n_{L}\right)$. Hence, in the following discussions, arithmetic operations on these variables are all in the context of "almost surely." Using Theorem 1 and AF-RAKE, we have

$$
\frac{\chi_{C}\left(n_{L}\right)}{\chi_{D}\left(n_{L}\right)}=\frac{r_{\text {coop }}^{2}}{r_{d}^{2}}= \begin{cases}1 & : 1 / 2<\rho<\sqrt{2} / 2 \\ \frac{\left(\lambda+\frac{\rho^{2}}{2 \sqrt{\rho^{2}-1 / 4}}+\frac{1}{2}\right)^{2}}{(\lambda+1)^{2}}: \sqrt{2} / 2 \leq \rho \leq \lambda\end{cases}
$$

One observation from (13) is that when the relay distance factor $\rho$ is less than $\sqrt{2} / 2$, collaborative relay does not result in extra interference. However, the tradeoff is that restricting the relay selection to such small area will limit the possibility of finding a relay. Finally, we recognize that the model simplification in Figure 5 and 6 can heavily impact this conclusion, at least the value of $\sqrt{2} / 2$. Nevertheless, this trend indicates the advantage of using relay nodes in close distance.

\footnotetext{
5"Almost surely" means that as $n$ goes to infinity the event happens with probability of 1 [30, Ch.6].
} 


\section{Effectiveness of Cooperative Relay}

When the amount of spectrum resource is fixed, using extra number of channels implies reduced per-channel resource. We use this metric to evaluate the penalty of elevated interference. Next, we integrate this penalty measure with the per-link relay gain to compare the overall system throughput with and without collaborative relays.

Let the system throughput $S$ be the sum of individual user throughput. Because each transmission is of identical length, the overall system throughput is the product of throughput per-resource and the number of transmissions.

$$
\begin{aligned}
S_{D T}\left(n_{L}\right) & =n_{L} \cdot C_{D T} \cdot \frac{B}{\chi_{D}\left(n_{L}\right)} \\
S_{A F-R A K E}\left(n_{L}\right) & =n_{L} \cdot C_{A F-R A K E} \cdot \frac{B}{\chi_{C}\left(n_{L}\right)} \\
S_{A F-F D D}\left(n_{L}\right) & =n_{L} \cdot C_{A F-R A K E} \cdot \frac{B}{2 \chi_{C}\left(n_{L}\right)}
\end{aligned}
$$

where $B$ represents the total amount of available resource, and $C_{D T}, C_{A F-R A K E}, C_{A F-F D D}$ represent the capacity of direct transmission, AF-RAKE and AF-FDD defined in Section II, respectively. As mentioned before, AF-FDD requires $2 \times$ channels compared to AF-RAKE.

For AF-RAKE based collaborative relays to be effective, i.e. having a higher system throughput compared to that of direct transmission, we require

$$
\frac{S_{A F-R A K E}\left(n_{L}\right)}{S_{D T}\left(n_{L}\right)} \geq 1
$$

Equivalently, the collaboration gain, defined as $\frac{C_{A F-R A K E}}{C_{D T}}$, needs to overcome the interference degradation, i.e.

$$
\frac{C_{A F-R A K E}}{C_{D T}} \geq \frac{\chi_{C}\left(n_{L}\right)}{\chi_{D}\left(n_{L}\right)}:=T_{A F-R A K E} .
$$

We hereby refer to $T_{A F-R A K E}$ as the critical ratio of AF-RAKE. Similarly for AF-FDD, we require

$$
\frac{C_{A F-F D D}}{C_{D T}} \geq \frac{2 \chi_{C}\left(n_{L}\right)}{\chi_{D}\left(n_{L}\right)}:=T_{A F-F D D}
$$

Substitute (13) into (16) and (17), we have

$$
\begin{aligned}
& T_{A F-R A K E}=T_{A F-T D D}=T_{D F} \\
& =\left\{\begin{array}{lll}
1 & : \quad 1 / 2<\rho<\sqrt{2} / 2 \\
\frac{\left(\lambda+\frac{\rho^{2}}{2 \sqrt{\rho^{2}-1 / 4}}+\frac{1}{2}\right)^{2}}{(\lambda+1)^{2}} & : & \sqrt{2} / 2 \leq \rho \leq \lambda
\end{array}\right.
\end{aligned}
$$


TABLE II

Numerical VAlues of CRitical Ratio $(\lambda=1)$

\begin{tabular}{|c|c|c|c|c|c|c|c|c|}
\hline & $\rho$ & $\leq \sqrt{2} / 2$ & 0.75 & 0.80 & 0.85 & 0.90 & 0.95 & 1 \\
\hline \multirow{2}{*}{$\begin{array}{c}\text { One Node } \\
\text { Relay }\end{array}$} & AF-RAKE & 1 & 1.0031 & 1.0124 & 1.0257 & 1.0416 & 1.0595 & 1.0788 \\
\cline { 2 - 9 } & AF-FDD & 2 & 2.0062 & 2.0249 & 2.0514 & 2.0833 & 2.1190 & 2.1577 \\
\hline $\begin{array}{c}\text { Multiple Nodes } \\
\text { Relay }\end{array}$ & AF-RAKE & 1 & 1.0599 & 1.1284 & 1.1962 & 1.2637 & 1.3315 & 1.3995 \\
\cline { 2 - 9 } & AF-FDD & 2 & 2.1198 & 2.2567 & 2.3923 & 2.5275 & 2.6629 & 2.7990 \\
\hline
\end{tabular}

$$
T_{A F-F D D}= \begin{cases}2 & : 1 / 2<\rho<\sqrt{2} / 2 \\ \frac{2\left(\lambda+\frac{\rho^{2}}{2 \sqrt{\rho^{2}-1 / 4}}+\frac{1}{2}\right)^{2}}{(\lambda+1)^{2}}: \sqrt{2} / 2 \leq \rho \leq \lambda\end{cases}
$$

Table II lists some numerical values for the critical ratio under different $\rho$. For example, when $\rho=\lambda=1, T_{A F-R A K E}=1.0788$ and $T_{A F-F D D}=2.1577$. This conclusion is different from those from single link transmissions where collaboration always benefits (see Section II). The critical ratio provides a guideline for designing collaborative relays on a single link basis.

\section{E. Extensions to Arbitrary Number of Relays}

The above conclusions assume each direct link is assisted by exactly one relay. When there are multiple relay nodes, the range of interference depends on the node that is furthermost from the source and destination. Under the constraint that each relay node needs to be within $r_{R}=\rho r_{C}$ from source and destination, the maximum interference generated by the collaborative relay is represented by a disk of radius

$$
d_{\max }= \begin{cases}(\lambda+1) r_{C} & : \quad 1 / 2<\rho<\sqrt{2} / 2 \\ \left(\lambda+\sqrt{\rho^{2}-1 / 4}+\frac{1}{2}\right) r_{C} & : \quad \sqrt{2} / 2 \leq \rho \leq \lambda\end{cases}
$$

Hence, the corresponding disk in unit disk graph is of radius $d_{\max } / 2$.

Such simplification allows us to approximate the maximum critical ratio $T_{\max }$ as following, which represents the critical ratio when the multiple cooperative relays are simultaneously chosen and placed in positions that lead to the maximum interference sensitive range.

$$
T_{\text {max }}:=\frac{\chi_{M A X}\left(n_{L}\right)}{\chi_{D}\left(n_{L}\right)}= \begin{cases}1 & : 1 / 2<\rho<\sqrt{2} / 2 \\ \frac{\left(\lambda+\sqrt{\rho^{2}-1 / 4}+1 / 2\right)^{2}}{(\lambda+1)^{2}}: \sqrt{2} / 2 \leq \rho \leq \lambda\end{cases}
$$


Intuitively, a direct link should use collaborative relays if the cooperation gain exceeds $T_{\max }$. For $\rho=\lambda=1 T_{\max }=1.3995$ if using AF-RAKE or AF-TDD, and $T_{\max }=2.7990$ if using AF-FDD or DF. Table II lists an additional set of results under various choices of $\rho$ and $\lambda=1$. However, this simplified analysis is based on the assumption that all the nodes still have the same interference-sensitive range. When there are multiple relays, the transmit power density changes, which will affect the interference-sensitive range $r_{I}$. In this case, we need to use the physical interference model to determine the relationship between $r_{I}$ and the number of relay nodes. We plan to address this in a future study.

\section{Vi. Simulation Results}

\section{A. Simulation Setup and Evaluation Metrics}

We perform simulations assuming noisy immobile radio networks, where each transmission experiences frequency flat Rayleigh fading. We focus on random network topologies by randomly deploying a set of nodes on a given area and examine the average results over 1000 deployments $^{6}$. We use a simple minimum-distance-idle-first strategy to select relay node, where each source node selects a nearest (to both source and destination) idle node as its relay. We focus on conflictfree transmissions and apply the algorithms in Section IV to assign channels to links but vary the priorities of direct and relay links. We consider several performance metrics.

- System throughput - The total system throughput based on both ergodic and outage capacities.

- System performance pain - An indicator on the throughput gain a collaborative relay should achieve in an isolated environment (without any impact of interference) to remain advantageous in an interference-oriented environment.

- Link blocking probability - The rate of direct link failures because of channel shortage. A direct link fails if it is not assigned with any channel.

- Relay usage probability - The probability of relay usage across all the active direct links. A relay is used if the relay link is assigned with a channel.

\footnotetext{
${ }^{6} \mathrm{We}$ also examined the performance under grid networks, with similar conclusions. The results are omitted due to space
} limitation. 
While the system throughput and performance gain measure the overall system performance and the superiority of collaborative relay, the link blocking probability measures the impact on individual user fairness.

Our simulations consider two scenarios. In the first scenario, we assume that the spectrum resource can be flexibly partitioned and per-channel resource depends on the total number of channels required for conflict-free transmissions. This allows us to verify the analytical findings developed in Section V. In the second scenario, we assume that the spectrum resource is prepartitioned into fixed number of channels, and apply the channel allocation schemes proposed in Section IV to examine the tradeoffs between throughput and fairness.

\section{B. Scenario 1: Flexible Channel Partitions}

We start with random networks with a fixed amount of spectrum but flexible channel partition. The system needs to partition spectrum into a set of channels so that each active link is assigned with exact one channel. We randomly deploy nodes in a unit disk area where each node has a communication range of $r_{C}=0.1$. In addition, we assume each link experiences Rayleigh flat fading with $\gamma=0 d B$ and $E\left(\left|h_{i j}\right|^{2}\right)=1$. We scale channel statistics following the propagation model defined in [21], with the path-loss factor $\alpha=2$. For each direct link, we randomly deploy one node within $r_{R}=\rho r_{C}$ distance from both source and destination as the relay node.

We first verify the analytical results in Section $\mathrm{V}$ in terms of the number of channels required to achieve conflict-free allocation. We compare the results of asymptotic analysis to those from simulations. Table III summarizes the results for both direct transmission (DT) and AF-RAKE, where EXP represents the simulation results and ANA represents the analytical results. In this example, we assume $\lambda=\rho=1$. When the number of links is large, the analytical results provide a reasonable approximation to the actual simulation results. More importantly, AF-RAKE requires more channels than that of DT, mapping to the penalty of elevated interference in collaborative relays.

Next, we examine the gain of collaborative relay in terms of system throughput. In Figure 7 we examine the system performance gain, defined by $S_{A F-R A K E}\left(n_{L}\right) / S_{D T}\left(n_{L}\right)$, using both ergodic and outage throughput at $\lambda=\rho=1$. When the number of links is small, i.e. less than 100 , the impact of interference is negligible and the system performance gain is similar to the single link throughput gain, i.e. 1.2 for ergodic throughput and 1.8 for outage throughput. As the number 
TABLE III

The Number of Channels Required to Achieve Conflict-Free Allocation

\begin{tabular}{|r|c|c|c|c|c|c|c|}
\hline No. of Links & 50 & 100 & 150 & 200 & 300 & 400 & 500 \\
\hline DT-EXP & 3.24 & 4.42 & 5.38 & 6.22 & 7.77 & 9.27 & 10.64 \\
\hline DT-ANA & 1.73 & 3.46 & 5.20 & 6.93 & 10.40 & 13.86 & 17.32 \\
\hline AF-EXP & 3.44 & 4.81 & 5.90 & 6.87 & 8.76 & 10.53 & 12.18 \\
\hline AF-ANA & 1.87 & 3.74 & 5.61 & 7.47 & 11.21 & 14.95 & 18.69 \\
\hline
\end{tabular}

of links increases, the system performance gain decreases as the impact of interference starts to dominate and penalizes the perform of collaborative relays. We also include the asymptotic gain based on the worst case scenario where each link pair is of $r_{C}$ apart. This estimation serves as an upper-bound to system performance gain under a large number of links.

In Figure 8, we examine the impact of relay distance factor $\rho$ assuming 300 links and $r_{C}=0.1$. As expected, increasing $\rho$ leads to system degradations. We note, however, the choice of $\rho^{2}$ directly impacts the probability of finding an available relay node, which is not captured in this simulation.

\section{Scenario 2: Fixed Channel Partitions}

Next, we examine the performance of the proposed channel allocation schemes in Section IV using random network topologies. We deploy 1000 nodes in a unit disk area and randomly activate links ${ }^{7}$. We divide the spectrum into a fixed number of channels, each of bandwidth $W=1$. We allocate channels to both direct and relay links to avoid conflict and maximize the number of links supported. Similarly, each node has a communication range of $r_{C}=0.1$.

Figure 9 shows the average system ergodic and outage throughput for direct transmissions (DT), AF-RAKE and AF-FDD, assuming two channels of identical statistics. We also compare the performance of collaborative and non-collaborative channel access, marked by CA and NCA respectively. We see that AF-RAKE significantly outperforms AF-FDD and DT in terms of both ergodic and outage throughput. Similarly to the observation in Figure 7, the overall system performance gain decreases with the number of links. In particular, for the outage throughput, the

\footnotetext{
${ }^{7}$ This simulation is different from those in previous section - there is no guarantee that each link can find an available relay.
} 


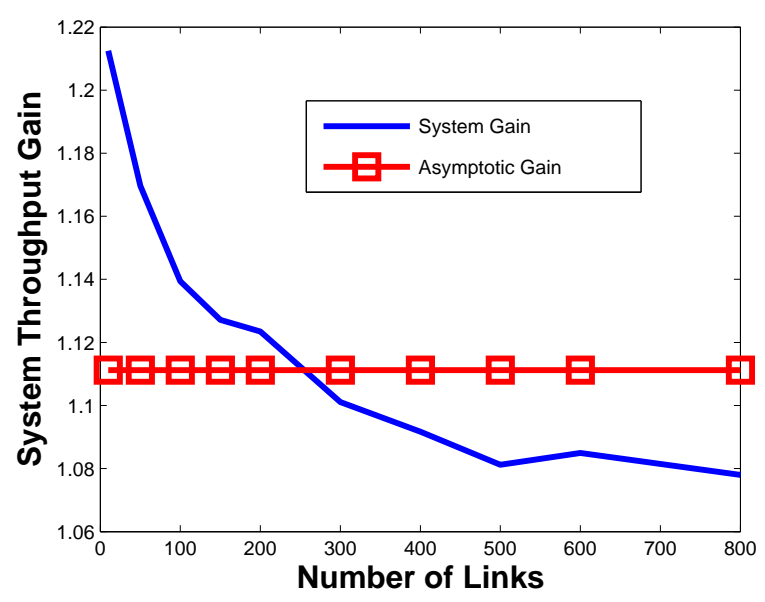

(a) Ergodic Throughput

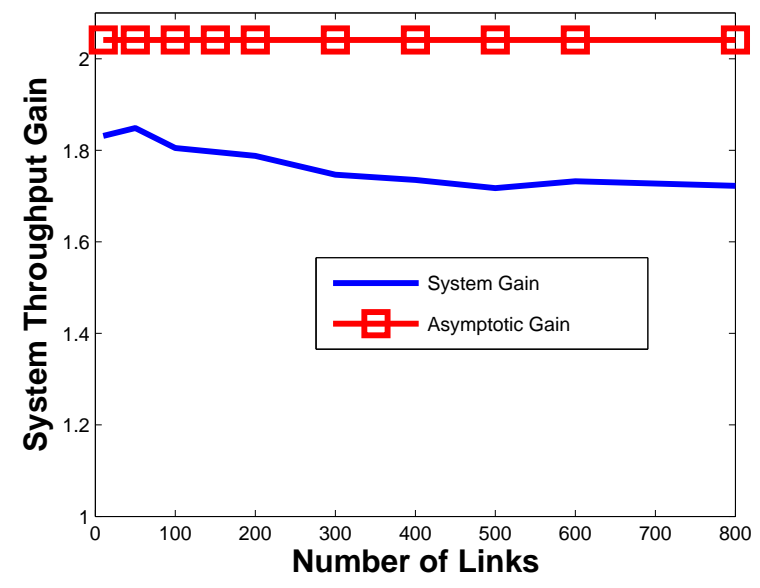

(b) Outage Throughput

Fig. 7. The effectiveness of collaborative relay in terms of system performance gain, assuming random networks, $\rho=\lambda=1$.

gain decreases from roughly $200 \%$ at 10 links to $160 \%$ at 300 links. We also observe that AFFDD outperforms DT significantly in outage throughput and marginally in ergodic throughput. This is because AF-FDD requires two channels per collaborative relay set, which diminishes the gain of single-link throughput.

One interesting observation is that NCA outperforms CA in outage throughput but not in ergodic throughput. This can be explained by the statistics in Figure 10. By prioritizing direct links over relay links, CA leads to lower probability of direct link failure at the cost of less relay usage. In the case of outage throughput, the loss due to reduced relay usage exceeds the gain 


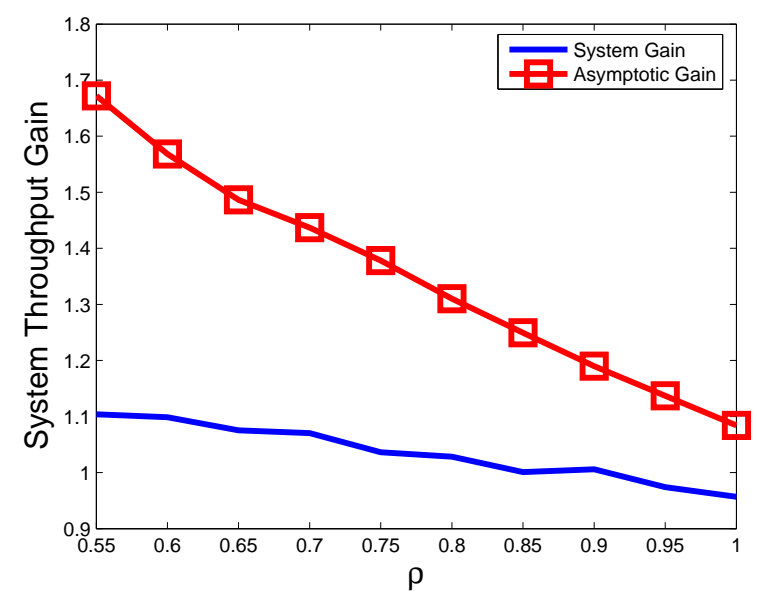

(a) Ergodic Throughput

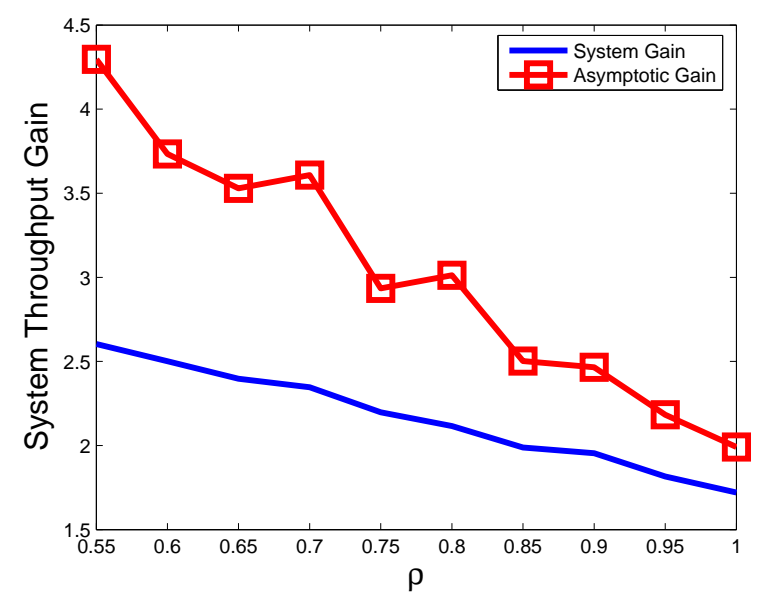

(b) Outage Throughput

Fig. 8. The effectiveness of collaborative relay in terms of system performance gain, assuming random networks, 300 links.

of supporting more direct links. Therefore, NCA based channel access outperforms CA in terms of outage throughput. In practice, the choice between CA and NCA depends on the application context: CA focuses on minimizing link blocking probability while NCA focuses on maximizing total system throughput.

Finally, we examine the impact of the amount of resource on system performance. We assume that each channel has bandwidth $W=1$ so that adding channels implies more spectrum. Figure 11 compares the system throughput of various schemes, assuming 160 active direct links in the network. As expected, increasing the number of channels significantly improves system 


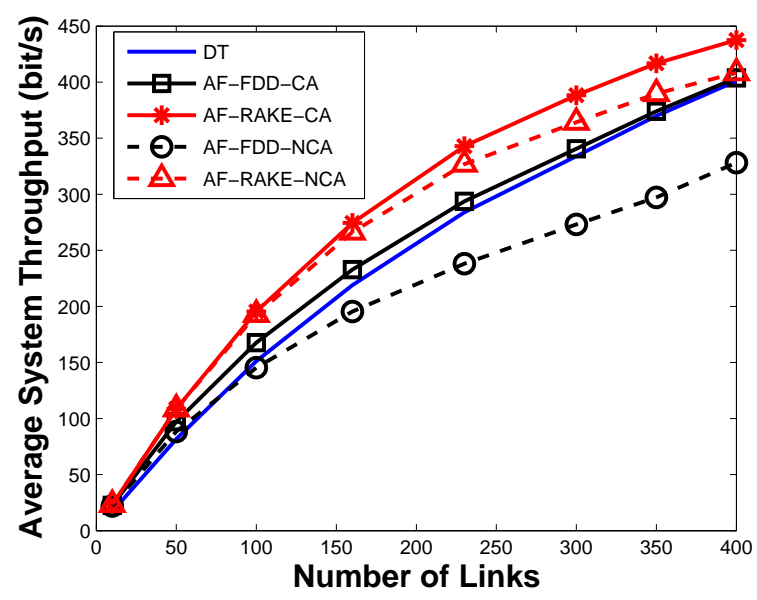

(a) Ergodic Throughput

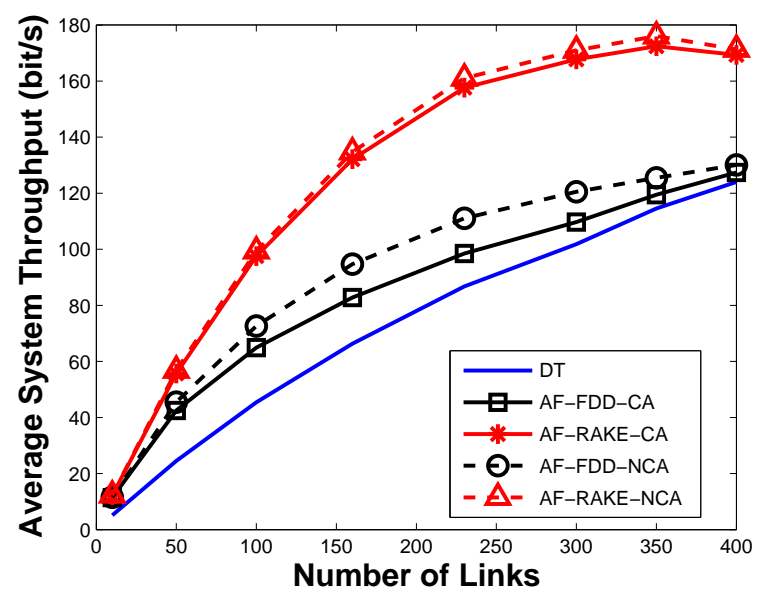

(b) Outage Throughput

Fig. 9. System ergodic throughput and outage throughput as a function of the number of links for two channels.

throughput, especially the outage throughput. Similarly, these observations can be explained by the statistics of direct link failure and relay usage in Figure 12. We note that given the number of active links, the performance of AF-FDD approaches that of AF-RAKE as the number of channels increases.

\section{DISCUSSIONS}

\section{A. Extension to Mobile Scenarios}

In this paper, we focus on static networks such as sensor or mesh networks. However, our work can be extended to mobile networks. In particular, because our analytical results assume 


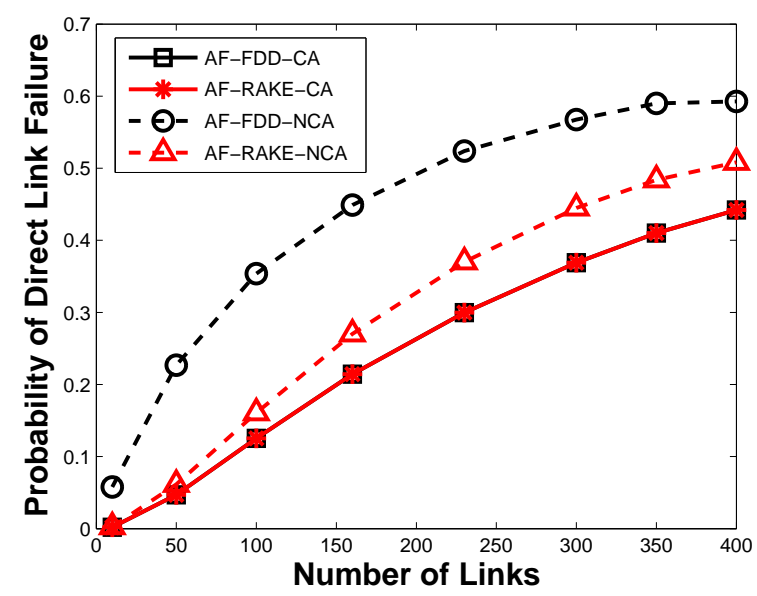

(a) Probability of direct link failure

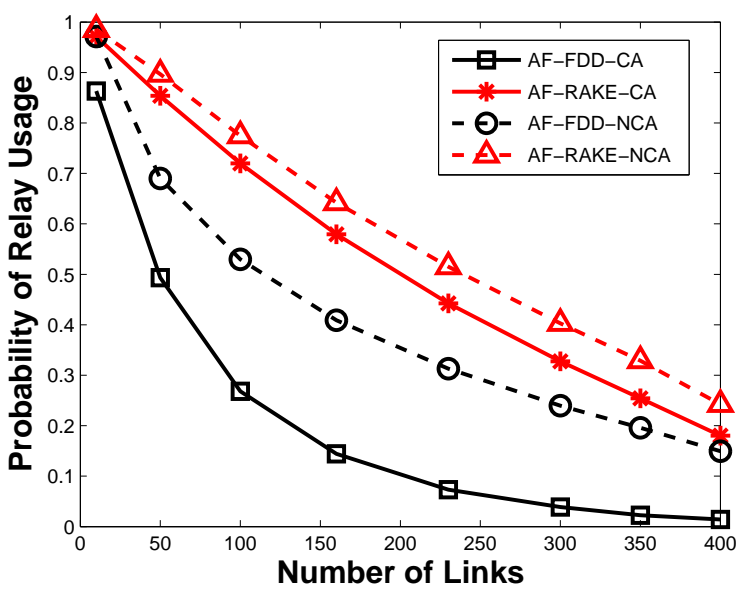

(b) Probability of relay usage

Fig. 10. Cooperation usage and link success statistics for systems with two channels

random networks with a fixed network density, the results can be easily applied to a large-scale mobile network where nodes move but maintain similar network density.

We also note that there are several existing solutions on distributed channel allocations where nodes apply local actions to tune network-wide channel allocations [26], [27]. Our channel allocation schemes can be extended to mobile networks by utilizing these distributed schemes. However, how to reliably identify conflicting neighbors remains as a challenging open research problem. 


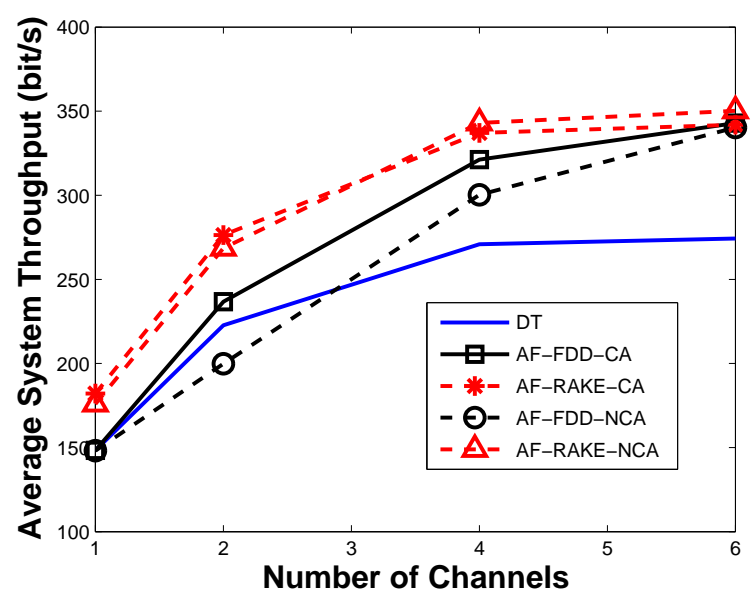

(a) Ergodic Throughput

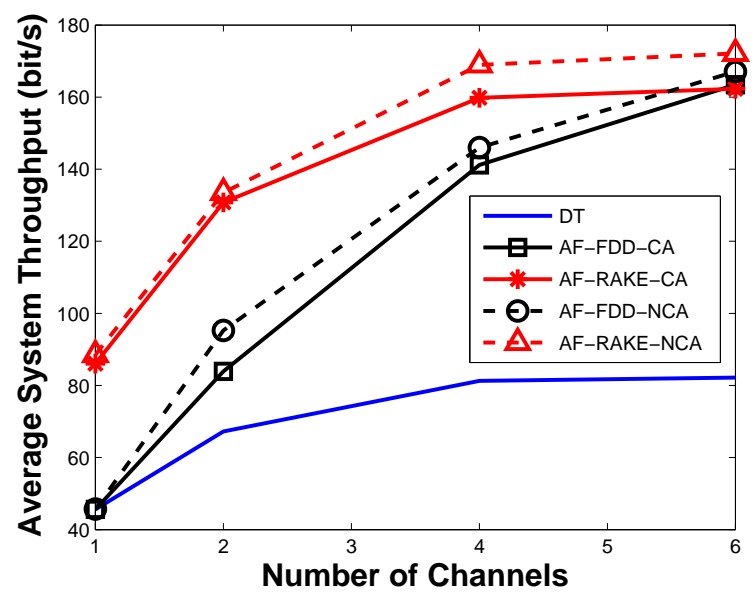

(b) Outage Throughput

Fig. 11. System ergodic throughput and outage throughput as a function of the number of channels assuming 160 active direct links.

\section{B. Extension to Other Relay Strategies}

In this paper we examine the performance of collaborative relays by using amplify-and-forward (AF) strategies. Our approach can be extended to other strategies by deriving the appropriate interference-sensitive range, and the link throughput in an isolated scenario. For example, the multi-source-multi-relay-multi-destination scheme in [11] requires more channels or time slots than a single source-destination-relay AF. As a result, the number of channels required for conflict-free transmissions will increase compared to that of AF, which leads to a higher critical ratio in terms of single-link throughput improvement. Overall, our analytical result provides a 


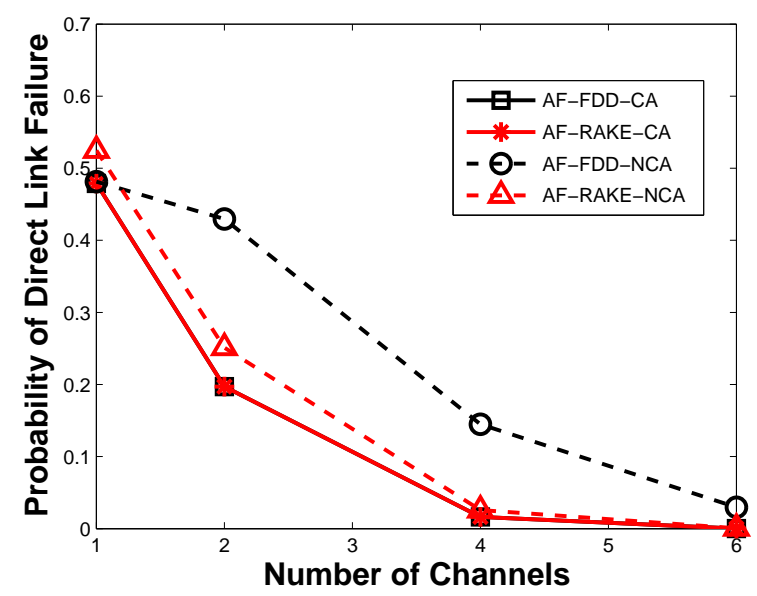

(a) Probability of direct link failure

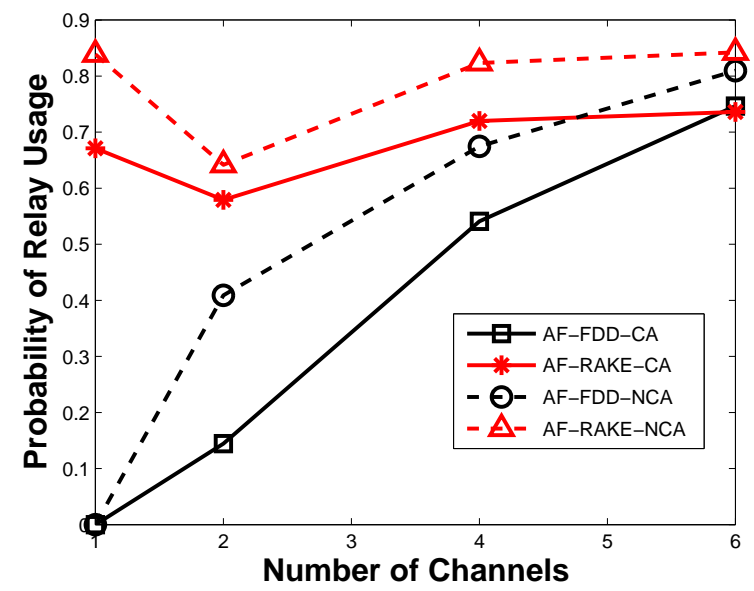

(b) Probability of relay usage

Fig. 12. Cooperation usage and link blocking statistics for variable numbers of channels and 160 links.

practical guideline to design and evaluate cooperative relay without delving into complex network optimization.

\section{Other Practical Issues}

In this paper, we use a simplified protocol implementation. In practice, the performance of collaborative relay also depends on the coordination overhead, level of synchronization, node mobility and channel access protocols. We are currently investigating the impact of these issues by implementing collaborative relays on 802.11 devices. 


\section{CONCLUSION}

In this paper, we examine the feasibility of applying collaborative relays to large-scale wireless networks for throughput improvement. While collaborative relays are effective in small isolated scenarios, its effectiveness in large-scale wireless networks is seriously penalized by an elevated level of interference. We show that asymptotically there exists a threshold, defined as the critical ratio, where the collaborative relay improves the network throughput only if the corresponding single-link throughput gain exceeds the critical ratio. Simulation results verify the asymptotic estimations. We also present two channel allocation mechanisms to mitigate interference and achieve conflict-free transmissions.

While our work provides an initial step towards deploying collaborative relay in a large wireless network, there are still many open problems. In this paper we only examine the performance of a limited set of collaborative relay strategies, focusing on the number of channels that a collaborative relay uses. In our future work, we plan to explore other relay strategies and their patterns of channel usage. Our analytical results are based on the protocol interference model, which is a pessimistic simplification of the physical interference model. We are currently extending our analysis to use the physical model and exploring the impact of power allocation and interference mitigation.

\section{ACKNOWLEDGMENT}

The authors would like to thank the reviewers and the Associate Editor for their insightful suggestions on the manuscript, which has helped to improve the paper significantly.

\section{REFERENCES}

[1] S. S. Barbarossa et al., "Cooperative wireless networks based on distributed space time coding," in Proc. of the International Workshop on Wireless Ad-hoc Networks, June 2004.

[2] J. N. Laneman, D. N. C. Tse, and G. W. Wornell, "Cooperative diversity in wireless networks: Efficient protocols and outage behavior," in IEEE Trans. on Information Theory, vol. 50, no. 12, December 2004, pp. 3062-3080.

[3] A. Sendonaris, E. Erkip, and B. Aazhang, "User cooperation diversity. part II: implementation aspects and performance analysis," in IEEE Trans. on Communications, vol. 51, no. 11, November 2003, pp. 1939-1948.

[4] A. H $\phi$ st-Madsen and J. Zhang, "Capacity bounds and power allocation for wireless relay channels," IEEE Trans. On Information Theory, vol. 51, no. 6, pp. 2020-2040, Jun. 2005.

[5] G. Kramer, M. Gastpar, and P. Gupta, "Cooperative strategies and capacity theorems for relay networks," in IEEE Trans. Inform. Theory, vol. 51, no. 9, September 2005, pp. 3037-3063. 
[6] K. Azarian, H. E. Gamal, and P. Schniter, "On the achievable diversity-multiplex tradeoff in half-duplex cooperative channels," IEEE Trans. on Information Theory, vol. 51, no. 12, pp. 4152-4172, Dec. 2005.

[7] X. Deng and A. M. Haimovich, "Power allocation for cooperative relaying in wireless networks," IEEE Communications Letters, vol. 9, no. 11, pp. 994-997, Nov. 2005.

[8] S. Serbetli and A. Yener, "Optimal power allocation for relay assisted f/tdma ad hoc networks," in Wireless Networks, Communications and Mobile Computing, 2005 International Conference on.

[9] A. Bletsas, A. Khisti, D. P. Reed, and A. Lippman, "A simple cooperative diversity method based on network path selection," IEEE Journal On Selected Areas In Communications, vol. 24, no. 3, pp. 659-672, Marth 2006.

[10] B. Zhao and M. C. Valenti, "Practical relay networks: A generalization of hybrib-ARQ," IEEE Journal On Selected Areas In Communications, vol. 23, no. 1, pp. 7-18, Jan. 2005.

[11] G. Li and H. Liu, "On the capacity of broadband relay networks," in Signals, Systems and Computers, 2004. Conference Record of the Thirty-Eighth Asilomar Conference on.

[12] — - "Resource allocation for OFDMA relay networks," in Signals, Systems and Computers, 2004. Conference Record of the Thirty-Eighth Asilomar Conference on.

[13] T. Abe, H. Shi, T. Asai, and H. Yoshino, "A relaying scheme for MIMO wireless networks with multiple source and destination pairs," in 2005 Asia-Pacific Conference on Communications, 2005.

[14] M. Qin and R. Blum, "Capacity of wireless ad hoc networks with cooperative diversity: a warning on the interaction of relaying and multi-hop routing," in IEEE International Conference on Communications, 2005.

[15] G. Scutari, S. Barbarossa, and D. Ludovici, "Cooperation diversity in multihop wireless networks using opportunistic driven multiple access," in Proc. of IEEE SPAWC, June 2003, pp. 170-174.

[16] A. Mercado and B. Azimi-Sadjadi, "Power efficient link for multi-hop wireless networks," in Proc. of the 41st Allerton Conference, October 2003.

[17] D. Tse and P. Viswanath, Fundamentals of Wireless Communications. Cambridge University Press, 2005.

[18] P. Gupta and P. Kumar, "The capacity of wireless networks," IEEE Transactions on Information Theory, vol. 46, no. 2, pp. 388-404, March 2000.

[19] A. Sahai, R. Tandra, and N. Hoven, "Opportunistic spectrum use for sensor networks: the need for local cooperation," 2006, submitted to Information Processing in Sensor Networks(IPSN).

[20] H. Ma, H. M. K. Alazemi, and S. Roy, "Stochastic model for optimizing physical carrier sensing and spatial reuse in wireless ad hoc networks," in Proc. of IEEE MASS 2005, November 2005.

[21] T. S. Rappaport, Wireless Communications: Principles and Practice, 2nd ed. Prentice Hall PTR, 2001.

[22] R. Diestel, Graph Theory. Springer-Verlag, Heidelberg, 2005.

[23] M. R. Garey and D. S. Johnson, Computers and Intractability: A Guide to the Theory of NP-Completeness. W. H. Freeman, 1990.

[24] S. Ramanathan, "A unified framework and algorithm for channel assignment in wireless networks," Wireless Networks, vol. 5, no. 2, pp. 81-94, March 1999.

[25] C. Peng, H. Zheng, and B. Y. Zhao, "Utilization and fairness in spectrum assignemnt for opportunistic spectrum access," Mobile Networks and Applications (MONET), 2006.

[26] L. Cao and H. Zheng, "Distributed spectrum allocation via local bargaining," in IEEE SECON, Santa Clara, CA, Sept. 2005.

[27] H. Zheng and L. Cao, "Device centric spectrum management," in Proc. of IEEE Dyspan, Baltimore, MD, November 2005. 
[28] M. Penrose, Random Geometric Graphs. Oxford University Press, 2003.

[29] C. McDiarmid, "Random channel assignment in the plane," Random Structures \& Algorithms, vol. 22, pp. 187-212, March 2003.

[30] K. B. Athreya and S. N. Lahiri, Measure Theory and Probability Theory. Springer-Verlag, 2006. 